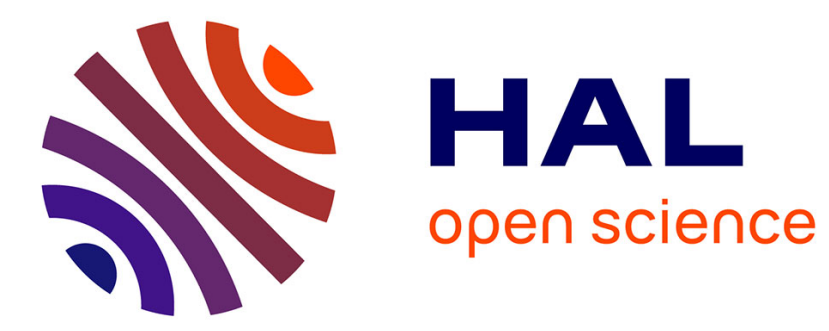

\title{
Quantum dynamics by the constrained adiabatic trajectory method
}

Arnaud Leclerc, Stéphane Guérin, Georges Jolicard, John P Killingbeck

\section{To cite this version:}

Arnaud Leclerc, Stéphane Guérin, Georges Jolicard, John P Killingbeck. Quantum dynamics by the constrained adiabatic trajectory method. Physical Review A : Atomic, molecular, and optical physics [1990-2015], 2011, 83 (3), 10.1103/PhysRevA.83.032113 . hal-00578514

\section{HAL Id: hal-00578514 https://hal.science/hal-00578514}

Submitted on $10 \mathrm{Jul} 2018$

HAL is a multi-disciplinary open access archive for the deposit and dissemination of scientific research documents, whether they are published or not. The documents may come from teaching and research institutions in France or abroad, or from public or private research centers.
L'archive ouverte pluridisciplinaire HAL, est destinée au dépôt et à la diffusion de documents scientifiques de niveau recherche, publiés ou non, émanant des établissements d'enseignement et de recherche français ou étrangers, des laboratoires publics ou privés. 


\title{
Quantum dynamics by the constrained adiabatic trajectory method
}

\author{
A. Leclerc, ${ }^{1, *}$ S. Guérin, ${ }^{2}$ G. Jolicard, ${ }^{1}$ and J. P. Killingbeck ${ }^{3}$ \\ ${ }^{1}$ Institut UTINAM, CNRS UMR 6213, Université de Franche-Comté, Observatoire de Besançon, 41 bis Avenue de l'Observatoire, \\ Boîte Postale 1615, F-25010 Besançon cedex, France \\ ${ }^{2}$ Laboratoire Interdisciplinaire Carnot de Bourgogne (CNRS UMR 5209, Université de Bourgogne), Boîte Postale 47870, \\ F-21078 Dijon, France \\ ${ }^{3}$ Centre for Mathematics, University of Hull, Hull HU6 7RX, United Kingdom
}

(Received 1 December 2010; published 16 March 2011)

\begin{abstract}
We develop the constrained adiabatic trajectory method (CATM), which allows one to solve the time-dependent Schrödinger equation constraining the dynamics to a single Floquet eigenstate, as if it were adiabatic. This constrained Floquet state (CFS) is determined from the Hamiltonian modified by an artificial time-dependent absorbing potential whose forms are derived according to the initial conditions. The main advantage of this technique for practical implementation is that the CFS is easy to determine even for large systems since its corresponding eigenvalue is well isolated from the others through its imaginary part. The properties and limitations of the CATM are explored through simple examples.
\end{abstract}

DOI: 10.1103/PhysRevA.83.032113

\section{INTRODUCTION}

Modern developments and applications of quantum mechanics often involve complex chemical and even biological systems driven by laser fields (see, for instance, Ref. [1]). Solving (numerically) the time-dependent Schrödinger equation (TDSE) for such time-dependent systems then becomes very time consuming and sometimes even impossible. Finding numerical simplifications is an active research. For instance, one can mention the multiconfiguration time-dependent Hartree algorithm [2].

Techniques that lead to an efficient propagation of a timedependent problem often involve the Floquet theory, which allows one to incorporate fast oscillations of the external field (for instance, such as the optical oscillations of a laser field) in an enlarged Hilbert space [3]. For instance, it permits an adiabatic separation between the fast field oscillation dynamics and the slow time modulation of the field envelope (adiabatic Floquet theory [4]). This Floquet technique can be used alternatively to treat the full time dependence of the field, which is referred to as the $\left(t, t^{\prime}\right)$ approach [5].

Relevant processes are most often expected to be described in a small subspace, often named active space, through effective Hamiltonians. In particular, one can mention the time-dependent wave operator theory as a tool to extract dynamical active spaces [6].

A few years ago, Jolicard et al. [7] proposed the constrained adiabatic trajectory method (CATM) for solving the TDSE for a time-dependent potential. Since we use a quantum mechanical approach, the trajectory studied in the CATM is not a classical one but rather a constrained path followed by the wave function as it develops in time in a composite Hilbert space, which we describe below. Here, we investigate that method extending it for an initial condition as a general superposition of states for a small system, and emphasizing its principal novel feature, the use of a complex absorbing potential, which is itself time dependent. The usual approach is to propagate the wave function in small time steps, with

\footnotetext{
*Arnaud.Leclerc@utinam.cnrs.fr
}

PACS number(s): 03.65.Aa, 02.60.Lj, 02.60.Cb, 02.70.Hm

the Hamiltonian considered as constant over each step [8,9]. The radically different approach of the CATM is to limit the time development to only one term in a Floquet expansion of the wave function, achieving this by a careful choice of the varying absorbing potential. The problem of integrating the TDSE then becomes that of finding one eigenvector of the system's Floquet Hamiltonian. The method has some affinities with the $\left(t, t^{\prime}\right)$ approach [5] but represents a modification and improvement of it. The method finds the wave function at regular grid points throughout the interaction period, the principal requirement being to work with a sufficient number of points to describe the time-varying Hamiltonian and to allow the stable use of fast Fourier transforms.

In brief, the technique requires the wave function corresponding to the dynamics to connect to a single Floquet state, referred to as a constrained Floquet state (CFS), through the use of an artificial absorbing potential (or optical potential). The second role of the absorbing potential is to dilate the Floquet spectrum isolating well the eigenvalue corresponding to the CFS from the other ones. Thus, in practice, one needs to find this CFS to determine the dynamics.

In Sec. II, on the basis of Ref. [7], we summarize the technique CATM with its corresponding Floquet representation and recall the result when the initial condition is a single eigenstate of the free system. In Sec. III, we extend the technique to a more general initial state, as a superposition of eigenstates of the free system. This is analyzed for a two-state system. A forthcoming paper will treat the case of systems of higher dimension. In Sec. IV, we give an analytic treatment of the effect of the absorbing potential on the Floquet spectrum for a two-state model. The numerical limitations of the method and its accuracy are analyzed in Sec. V. We study examples with two- and three-level models, which illustrate the dual role played by the optical potential in Sec. VI. Section VII is devoted to the conclusion.

\section{The CATM}

\section{A. The Floquet representation}

We assume a system of Hamiltonian $H(q, t)$ (where the quantum coordinates have been denoted by $q$ ) defined in a 
basis $\{|j\rangle\}$. This Hamiltonian can usually be decomposed as $H(q, t)=H_{0}(q)+W(q, t)$ featuring a free system $H_{0}(q)$ subjected to an external time-dependent field corresponding to the interaction potential $W(q, t)$. In that case, $\{|j\rangle\}$ corresponds to the states of the free system. We assume that the interaction potential $W(q, t)$ acts on a duration $t \in[0, T]$ referred to as the physical duration in the following. We define an extra time interval $\left[T, T^{\prime}\right]$ after the physical interaction time during which (i) we add an artificial time-dependent absorbing (or optical) potential $\mathcal{V}(q, t)$ satisfying $\mathcal{V}(q, 0 \leqslant t \leqslant T)=\mathcal{V}\left(q, T^{\prime}\right)=0$, and (ii) we continuously extend the interaction such that $W\left(q, T^{\prime}\right)=W(q, 0)$. This construction features a periodic Hamiltonian $H\left(q, T^{\prime}\right)=H(q, 0)$.

Thus, we can define the corresponding Floquet Hamiltonian (or quasienergy operator) on the extended Hilbert space (product of the original Hilbert space, i.e., associated with the free system, by the space of $T^{\prime}$-periodic functions) [4]:

$$
H_{F}(q, t)=H_{0}(q)+W(q, t)+\mathcal{V}(q, t)-i \hbar \frac{\partial}{\partial t} .
$$

We consider the entire duration of the interaction + absorbing potential as a fundamental period $T^{\prime}\left(\omega_{0}=2 \pi / T^{\prime}\right)$, contrary to the traditional Floquet scheme in which $T^{\prime}$ is associated with the period of an external field (such as the optical period of a laser field). The Floquet states can be indexed with a double labeling $j, n$ linked to a finite-basis representation of the decoupled parts of Eq. (1), i.e., to the free-system eigenstates $(j \leftrightarrow|j\rangle)$ and to the operator $-i \hbar \partial_{t}$ (corresponding here to a Fourier basis, $\left.n \leftrightarrow|n\rangle \equiv\left|e^{-i n \omega_{0} t}\right\rangle\right)$. Thus, a complete basis is formed with the eigenstates $\left\{\left|\lambda_{j, n}(q, t)\right\rangle\right\}$ of $H_{F}$ :

$$
H_{F}\left|\lambda_{j, n}(q, t)\right\rangle=\hbar \omega_{\lambda_{j, n}}\left|\lambda_{j, n}(q, t)\right\rangle .
$$

Using the periodicity properties of the Floquet theorem $\left(\left|\lambda_{j, n}(q, t)\right\rangle=\left|\lambda_{j, 0}(q, t)\right\rangle e^{i n \omega_{0} t}, \omega_{\lambda_{j, n}}=\omega_{\lambda_{j, 0}}+n \omega_{0}\right)$, we can rigorously expand the solution of the TDSE with an initial limitation to the first Brillouin zone [3],

$$
|\Psi(q, t)\rangle=\sum_{j}\left\langle\lambda_{j, 0}(q, 0) \mid \Psi(q, 0)\right\rangle e^{-i \omega_{\lambda_{j, 0}} t}\left|\lambda_{j, 0}(q, t)\right\rangle .
$$

(Here, for simplicity, we only consider a bound spectrum, which, however, can feature imaginary parts; the extension to a system with a bound and continuous spectrum is, in principle, direct assuming a discretization of the continua). Usually, a great number of $\left|\lambda_{j, 0}\right\rangle$ is necessary to reconstruct $|\Psi(q, t)\rangle$. An interesting practical application of Eq. (3) is the development of a very reduced number of Floquet vectors and, in the best case of only one, which is the key idea of the CATM.

The method developed in this paper deals with the case of a single CFS which is labeled $j=\ell$ in the expansion (3). In this case, the CFS has to match, when projected at $t=0$, with the initial boundary conditions required for the wave function $\Psi(t=0)$ :

$$
\left\langle\lambda_{j, 0}(q, 0) \mid \Psi(q, 0)\right\rangle=\delta_{j, \ell},
$$

i.e.,

$$
|\Psi(q, t)\rangle=e^{-i \omega_{\lambda} t}|\lambda(q, t)\rangle,
$$

where we have omitted, in the latter, the index $\ell$ for simplicity: $\omega_{\lambda} \equiv \omega_{\lambda_{\ell, 0}},|\lambda(q, t)\rangle \equiv\left|\lambda_{\ell, 0}(q, t)\right\rangle$.
We will show below that, in practice, we do not get the exact equality (5) but a proportionality through a well-defined complex phase.

\section{B. Initial condition as an eigenstate of the free system}

Jolicard et al. [7] provided the matching with the initial condition for an initial state equal to a single state $|i\rangle$ of the $\{|j\rangle\}$ basis, i.e., $|\Psi(q, 0)\rangle=|i\rangle$. The connection between the Floquet eigenstate and the required initial state is made thanks to the addition of the absorbing potential $\mathcal{V}$ on the extra interval $\left[T, T^{\prime}\right]$. Below, we summarize this scheme and extend it in Sec. III to any required initial condition for the particular case of a two-state system.

In order to satisfy Eq. (5) (with a proportionality instead of the equality), it is sufficient to have the connection at $t=0$ :

$$
|\lambda(q, 0)\rangle \propto|i\rangle
$$

We remark that $|\lambda(q, t)\rangle$ is a Floquet vector of the extended Hilbert space, but fixing $t$ to a specific value leads to a component of this vector of dimension of the original Hilbert space. Equation (6) suggests the use of the following form for the absorbing potential:

$$
\mathcal{V}(t)=\sum_{j \neq i}-i V_{\mathrm{opt}}(t)|j\rangle\langle j|,
$$

with $V_{\text {opt }}(t)$ zero over $[0, T]$ and positive over [ $\left.T, T^{\prime}\right]$. As shown in Ref. [7], provided that

$$
\frac{1}{\hbar} \int_{T}^{T^{\prime}} V_{\mathrm{opt}}(t) d t \gg\left|\operatorname{Im}\left(\omega_{\lambda}\right)\right|\left(T^{\prime}-T\right),
$$

we can be sure that all channels except $|i\rangle$ are absorbed and that Eq. (6) is satisfied to a good approximation (as will be tested in Sec. VI).

\section{EXTENSION OF THE CATM TO A GENERAL INITIAL CONDITION: THE TWO-STATE CASE}

If we wish to work with the CATM in the case of an initial condition as a state superposition of the free system, i.e.,

$$
|\Psi(0)\rangle=\sum_{j} c_{j}|j\rangle
$$

then simple forms as Eq. (7) no longer work. (From now on, we do not explicitly write the dependence on the $q$ coordinates.)

Below, we provide the relevant absorbing potential that should be used for a two-state system of the Hamiltonian,

$$
H(t)=\hbar\left(\begin{array}{ll}
\Delta_{1}(t) & \Omega(t) \\
\Omega^{*}(t) & \Delta_{2}(t)
\end{array}\right)
$$

We consider the most general case with diagonal terms that are time dependent (due to Stark shifts of the states, for instance) and complex (i.e., including their lifetime). We assume that the coupling $\Omega(t)$ is, in general, different from zero only over the physical time interval $[0, T]$. During the extra time interval, the diagonal terms have to be continuously varied such that they recover their initial value in order to guarantee the periodicity: $\Delta_{j}\left(T^{\prime}\right)=\Delta_{j}(0)$. 
We show below that it is possible to treat any initial condition by adding the following absorbing potential over the supplementary interval $\left[T, T^{\prime}\right]$ :

$$
\begin{aligned}
& \mathcal{V}(t)=-i V_{\mathrm{opt}}(t)\left(\begin{array}{cc}
0 & 0 \\
-\frac{c_{2} e^{i} T_{t}^{T^{\prime}} \Delta_{2}\left(t^{\prime}\right) d t^{\prime}}{c_{1} e^{i \int_{t}^{T^{\prime}} \Delta_{1}\left(t^{\prime}\right) d t^{\prime}}} & 1
\end{array}\right), \\
& \text { with } \left.\quad V_{\text {opt }}(t)>0, \quad \forall t \in\right] T, T^{\prime}[\text {, } \\
& V_{\text {opt }}(t)=0, \quad \forall t \in[0, T] .
\end{aligned}
$$

The operator

$$
\Pi(t)=\left(\begin{array}{cc}
0 & 0 \\
-\frac{c_{2} e^{i} \int_{t}^{T^{\prime}} \Delta_{2}\left(t^{\prime}\right) d t^{\prime}}{c_{1} e^{i \int_{t}^{T^{\prime}} \Delta_{1}\left(t^{\prime}\right) d t^{\prime}}} & 1
\end{array}\right)
$$

involved in this definition (11) is a nonorthogonal (i.e., nonself-adjoint) projector, i.e., $\Pi^{2}=\Pi$, whose kernel is the initial state up to a phase correction:

$$
\Pi(t)\left(\begin{array}{l}
c_{1} e^{i \int_{t}^{T^{\prime}} \Delta_{1}\left(t^{\prime}\right) d t^{\prime}} \\
c_{2} e^{i \int_{t}^{T^{\prime}} \Delta_{2}\left(t^{\prime}\right) d t^{\prime}}
\end{array}\right)=0 .
$$

For this case, it is indeed possible to obtain the analytical asymptotic form of the Floquet eigenvector over the extra interval $\left[T, T^{\prime}\right]$, where the Hamiltonian contains just the free-system Hamiltonian and the absorbing potential. With the above definition and writing Floquet components $\langle j \mid \lambda(t)\rangle=$ $\lambda_{j}(t)$, one must solve the following system on $\left[T, T^{\prime}\right]$ :

$$
\begin{gathered}
\frac{\partial \lambda_{1}(t)}{\partial t}=i\left[\omega_{\lambda}-\Delta_{1}(t)\right] \lambda_{1}(t) \\
\frac{\partial \lambda_{2}(t)}{\partial t}=\frac{V_{\mathrm{opt}}(t)}{\hbar} \frac{c_{2} e^{i \int_{t}^{T^{\prime}} \Delta_{2}\left(t^{\prime}\right) d t^{\prime}}}{c_{1} e^{i \int_{t}^{T^{T^{\prime}}} \Delta_{1}\left(t^{\prime}\right) d t^{\prime}}} \lambda_{1}(t) \\
-\left(\frac{V_{\mathrm{opt}}(t)}{\hbar}-i\left[\omega_{\lambda}-\Delta_{2}(t)\right]\right) \lambda_{2}(t)
\end{gathered}
$$

The first component follows an exponential law: $\lambda_{1}(t)=$ $\lambda_{1}(T) \exp \left\{i\left[\omega_{\lambda}(t-T)-\int_{T}^{t} \Delta_{1}\left(t^{\prime}\right) d t^{\prime}\right]\right\}$. This function can be introduced in the second equation, and making use of the identity $\int_{T}^{t} V_{\text {opt }}\left(t^{\prime}\right) \exp \left[\frac{1}{\hbar} \int_{T}^{t^{\prime}} V_{\text {opt }}\left(t^{\prime \prime}\right) d t^{\prime \prime}\right] d t^{\prime}=$ $\hbar\left\{\exp \left[\frac{1}{\hbar} \int_{T}^{t} V_{\mathrm{opt}}\left(t^{\prime}\right) d t^{\prime}\right]-1\right\}$, we find

$$
\begin{aligned}
\lambda_{2}(t)= & \lambda_{2}(T) \exp \left\{i\left[\omega_{\lambda}(t-T)-\int_{T}^{t} \Delta_{2}\left(t^{\prime}\right) d t^{\prime}\right]\right\} \\
& \times \exp \left[-\frac{1}{\hbar} \int_{T}^{t} V_{\mathrm{opt}}\left(t^{\prime}\right) d t^{\prime}\right] \\
& +\frac{c_{2}}{c_{1}} \lambda_{1}(T) e^{i \omega_{\lambda}(t-T)} e^{i \int_{T^{\prime}}^{T} \Delta_{1}\left(t^{\prime}\right) d t^{\prime}} e^{i \int_{t}^{T^{\prime}} \Delta_{2}\left(t^{\prime}\right) d t^{\prime}} \\
& \times\left\{1-\exp \left[-\frac{1}{\hbar} \int_{T}^{t} V_{\mathrm{opt}}\left(t^{\prime}\right) d t^{\prime}\right]\right\} .
\end{aligned}
$$

Taking the $\lambda$ periodicity $\lambda_{j}\left(T^{\prime}\right) \equiv \lambda_{j}(0)$ into account, we obtain

$$
\begin{aligned}
\frac{\lambda_{2}(0)}{\lambda_{1}(0)}= & \frac{\lambda_{2}(T)}{\lambda_{1}(T)} \exp \left[-\frac{1}{\hbar} \int_{T}^{T^{\prime}} V_{\mathrm{opt}}(t) d t\right] \\
& \times \exp \left\{i \int_{T}^{T^{\prime}}\left[\Delta_{1}(t)-\Delta_{2}(t)\right] d t\right\} \\
& +\frac{c_{2}}{c_{1}}\left\{1-\exp \left[-\frac{1}{\hbar} \int_{T}^{T^{\prime}} V_{\mathrm{opt}}(t) d t\right]\right\},
\end{aligned}
$$

which, in the limits,

$$
\begin{gathered}
\frac{1}{\hbar} \int_{T}^{T^{\prime}} V_{\mathrm{opt}}(t) d t \gg 1, \\
\frac{1}{\hbar} \int_{T}^{T^{\prime}} V_{\mathrm{opt}}(t) d t \gg \int_{T}^{T^{\prime}}\left\{\operatorname{Im}\left[\Delta_{2}(t)\right]-\operatorname{Im}\left[\Delta_{1}(t)\right]\right\} d t,
\end{gathered}
$$

and for $\lambda_{2}(T)$ and $\lambda_{1}(T)$ of the same order, leads to

$$
\frac{\lambda_{2}(0)}{\lambda_{1}(0)} \rightsquigarrow \frac{c_{2}}{c_{1}} .
$$

We remark that, denoting the state vector of the original $\operatorname{TDSE}|\Psi(t)\rangle \equiv\left(\begin{array}{l}a_{1}(t) \\ a_{2}(t)\end{array}\right)$, the connection to a single Floquet vector (5) leads to $\lambda_{2}(T) / \lambda_{1}(T)=a_{2}(T) / a_{1}(T)$, i.e., to the ratio of the amplitude at the end of the process. If this ratio becomes very large, which corresponds to the specific case of an efficient population transfer to state 2 , the condition (17b) is not sufficient. In general, it should be replaced by the condition:

$$
\begin{aligned}
\frac{1}{\hbar} \int_{T}^{T^{\prime}} V_{\mathrm{opt}}(t) d t \gg & \int_{T}^{T^{\prime}}\left\{\operatorname{Im}\left[\Delta_{2}(t)\right]-\operatorname{Im}\left[\Delta_{1}(t)\right]\right\} d t \\
& +\ln \left[\left|a_{2}(T)\right|\right]-\ln \left[\left|a_{1}(T)\right|\right] .
\end{aligned}
$$

This is discussed in more detail in Sec. V B.

For an initial condition as a single state of the free system, i.e., $c_{2}=0, c_{1}=1$, one recovers $\lambda_{2}(0) \ll \lambda_{1}(0)$ [7]. In this case, we must note that conditions (17) are less restrictive than condition (8). This is due to the fact that conditions (17) are obtained constraining a ratio of two components, whereas, in Ref. [7], we wished to absorb the components, with an error lower than the computer accuracy. If the conditions (17) are satisfied, then we can force any eigenstate $|\lambda\rangle$ to obey the final condition

$$
\begin{aligned}
& \lambda_{1}(0)=\lambda_{1}(T) \exp \left\{i\left[\omega_{\lambda}\left(T^{\prime}-T\right)-\int_{T}^{T^{\prime}} \Delta_{1}(t) d t\right]\right\}, \\
& \lambda_{2}(0) \rightsquigarrow \lambda_{1}(T) \exp \left\{i\left[\omega_{\lambda}\left(T^{\prime}-T\right)-\int_{T}^{T^{\prime}} \Delta_{1}(t) d t\right]\right\} \frac{c_{2}}{c_{1}} .
\end{aligned}
$$

Thus, apart from a global constant $\lambda_{1}(T)$ that results from the diagonalization procedure, an exponentially decreasing term and a global phase, we obtain

$$
|\lambda(t=0)\rangle \propto|\psi(t=0)\rangle .
$$

This approximate proportionality is sufficient to impose the required initial connection to the Floquet eigenvector (6). This will be illustrated by an example given in Sec. VI. 


\section{ISOLATING ONE EIGENVALUE IN THE FLOQUET SPECTRUM}

The second role of the absorbing potential is to dilate the Floquet spectrum and so isolate the connected eigenvalue $\hbar \omega_{\lambda}$ [i.e., the one associated with the eigenvector $|\lambda(t)\rangle$ connected to the initial condition] from the other eigenvalue [denoted $\hbar \omega_{\lambda^{\prime}}$ associated with $\left.\left|\lambda^{\prime}(t)\right\rangle\right]$. For simplicity, we consider the initial condition as a single bound state $|1\rangle$ of $H_{0}$. The absorbing potential takes the form set out in Eq. (7).

We start connecting the solution $|\Psi(q, t)\rangle$ to the Floquet vector. This is achieved by solving the stationary problem (in the first Brillouin zone):

for $t \in[0, T]:$

$$
\left[-i \frac{\partial}{\partial t}+\left(\begin{array}{cc}
\Delta_{1}(t) & \Omega(t) \\
\Omega^{*}(t) & \Delta_{2}(t)
\end{array}\right)\right]|\lambda(t)\rangle=\omega_{\lambda}|\lambda(t)\rangle,
$$

for $t \in\left[T, T^{\prime}\right]:$

$$
\left[-i \frac{\partial}{\partial t}+\left(\begin{array}{cc}
\Delta_{1}(t) & 0 \\
0 & \Delta_{2}(t)-\frac{i}{\hbar} V_{\mathrm{opt}}(t)
\end{array}\right)\right]|\lambda(t)\rangle=\omega_{\lambda}|\lambda(t)\rangle .
$$

In the region $t \in\left[T, T^{\prime}\right]$, from Eq. (22b), we obtain (see Sec. III):

$$
\begin{gathered}
\lambda_{1}(t)=\lambda_{1}(T) \exp \left\{i\left[\omega_{\lambda}(t-T)-\int_{T}^{t} \Delta_{1}\left(t^{\prime}\right) d t^{\prime}\right]\right\}, \\
\lambda_{2}(t)=\lambda_{2}(T) \exp \left\{i\left[\omega_{\lambda}(t-T)-\int_{T}^{t} \Delta_{2}\left(t^{\prime}\right) d t^{\prime}\right]\right\} \\
\times \exp \left[-\frac{1}{\hbar} \int_{T}^{t} V_{\mathrm{opt}}\left(t^{\prime}\right) d t^{\prime}\right] .
\end{gathered}
$$

\section{A. Decoupled channels}

The situation is the easiest to follow in the elementary case in which the channels are not coupled $[\Omega(t)=0]$ and with constant diagonal terms $\Delta_{i}$. Thus, we make the instant $T$ coincide with $t=0$, to study the influence of the optical potential alone on the interval $\left[T=0, T^{\prime}\right]$ without any physical coupling terms. In this particular case, with $T=0$ and $t=T^{\prime}$, the previous system becomes

$$
\begin{gathered}
\lambda_{1}\left(T^{\prime}\right)=\lambda_{1}(0) e^{i\left(\omega_{\lambda}-\Delta_{1}\right) T^{\prime}} \\
\lambda_{2}\left(T^{\prime}\right)=\lambda_{2}(0) e^{i\left(\omega_{\lambda}-\Delta_{2}\right) T^{\prime}} \exp \left[-\frac{1}{\hbar} \int_{0}^{T^{\prime}} V_{\mathrm{opt}}\left(t^{\prime}\right) d t^{\prime}\right] .
\end{gathered}
$$

The same equations can be written for the other eigenstate $\left|\lambda^{\prime}\right\rangle$. Floquet eigenvectors must be periodic, i.e., $\lambda_{i}\left(T^{\prime}\right)=$ $\lambda_{i}(0)$. Thus, each Floquet eigenvalue simultaneously must satisfy two conditions:

$$
\begin{gathered}
1=e^{i\left(\omega_{\lambda}-\Delta_{1}\right) T^{\prime}}, \quad \text { if } \lambda_{1}(0) \neq 0, \\
1=\exp \left\{i\left[\left(\omega_{\lambda}-\Delta_{2}\right) T^{\prime}+\frac{i}{\hbar} \int_{0}^{T^{\prime}} V_{\mathrm{opt}}(t) d t\right]\right\}, \quad \text { if } \lambda_{2}(0) \neq 0 .
\end{gathered}
$$

The only solution is to have only one nonzero component for each eigenvector:

$$
\begin{aligned}
& \lambda_{1}(0) \neq 0 \quad \text { and } \quad \lambda_{2}(0)=0 \text {, i.e., } \omega_{\lambda}=\Delta_{1}, \\
& \lambda_{1}^{\prime}(0)=0 \quad \text { and } \quad \lambda_{2}^{\prime}(0) \neq 0 \text {, } \\
& \text { i.e., } \\
& \omega_{\lambda^{\prime}}=\Delta_{2}-\frac{i}{\hbar T^{\prime}} \int_{0}^{T^{\prime}} V_{\mathrm{opt}}(t) d t .
\end{aligned}
$$

The terms $\frac{2 k \pi}{T^{\prime}}$ are not mentioned because we work in a given Brillouin zone. In this simpliest case, the extension to an $N$-dimensional system is straightforward: All the eigenvalues connected to absorbed channels possess an imaginary term proportional to $\frac{1}{T^{\prime}} \int_{0}^{T^{\prime}} V_{\text {opt }}(t) d t$. Thus, we expect to obtain a dispersion of the eigenvalues in the complex plane, which will leave the other eigenvalues distant from the connected eigenvalue $\omega_{\lambda}$.

\section{B. General case}

In the present case of a two-level coupled system described by Eq. (22), it is possible to go further in the analytical description. In the region $t \in[0, T]$, one can rewrite Eq. (22a) as

$$
\left[-i \frac{\partial}{\partial t}+\left(\begin{array}{cc}
\Delta_{1}(t) & \Omega(t) \\
\Omega^{*}(t) & \Delta_{2}(t)
\end{array}\right)\right]|\lambda(t)\rangle e^{-i \omega_{\lambda} t}=0
$$

that is, as the same form of the original TDSE of solution $|\Psi(t)\rangle \equiv\left(\begin{array}{l}a_{1}(t) \\ a_{2}(t)\end{array}\right)$. We connect the two solutions invoking the initial conditions $a_{1}(0)=1, a_{2}(0)=0$, and $\lambda_{1}(0)=$ $\lambda_{1}(T) \exp \left\{i\left[\omega_{\lambda}\left(T^{\prime}-T\right)-\int_{T}^{T^{\prime}} \Delta_{1}(t) d t\right]\right\}, \lambda_{2}(0) \simeq 0$ (from Sec. II):

$$
\begin{aligned}
& \left(\begin{array}{l}
a_{1}(t) \\
a_{2}(t)
\end{array}\right) \lambda_{1}(T) \exp \left\{i\left[\omega_{\lambda}\left(T^{\prime}-T\right)-\int_{T}^{T^{\prime}} \Delta_{1}\left(t^{\prime}\right) d t^{\prime}\right]\right\} \\
& =\left(\begin{array}{l}
\lambda_{1}(t) \\
\lambda_{2}(t)
\end{array}\right) e^{-i \omega_{\lambda} t}
\end{aligned}
$$

The latter equation is just the proof of the Floquet theorem for our specific two-state problem. Considering the final physical time $t=T$, we get

$$
a_{1}(T)=e^{i \int_{T}^{T^{\prime}} \Delta_{1}(t) d t} e^{-i \omega_{\lambda} T^{\prime}},
$$

that is, we connect the imaginary part of the eigenvalue $\omega_{\lambda}$ to the final probability amplitude:

$$
\operatorname{Im}\left(\omega_{\lambda}\right)=\frac{1}{T^{\prime}} \int_{T}^{T^{\prime}} \operatorname{Im}\left[\Delta_{1}(t)\right] d t+\frac{1}{T^{\prime}} \ln \left[\left|a_{1}(T)\right|\right] .
$$

To get the counterpart relation for the other (nonconnected) eigenvalue $\omega_{\lambda^{\prime}}$, we reformulate the complete calculation with the adjoint of $H_{F}(t)$ (using $\partial_{t}^{\dagger}=-\partial_{t}$ ),

$$
H_{F}^{\dagger}(t)=H_{0}^{\dagger}+W^{\dagger}(t)+\mathcal{V}^{\dagger}(t)-i \hbar \frac{\partial}{\partial t},
$$

of eigenstates $\left\{\left|\widetilde{\lambda}_{j, n}(t)\right\rangle\right\}$,

$$
H_{F}^{\dagger}\left|\tilde{\lambda}_{j, n}(t)\right\rangle=\hbar \omega_{\lambda_{j, n}^{*}}^{*}\left|\tilde{\lambda}_{j, n}(t)\right\rangle,
$$


where $(\cdot)^{*}$ denotes the complex conjugate. For real energies of $H_{0}$ and real elements in $W(t)$, this latter equation corresponds to the same original problem as before but with the use of an exponentially diverging potential $\mathcal{V}^{\dagger}(t)$. Then, we have for the components of $\left|\tilde{\lambda}^{\prime}(t)\right\rangle$ [denoted as the eigenvector associated with the eigenvalue $\hbar \omega_{\lambda^{\prime}}^{*},\left|\tilde{\lambda}^{\prime}(t)\right\rangle$ is different from $\left|\lambda^{\prime}(t)\right\rangle$ in general]:

$$
\begin{aligned}
\tilde{\lambda}_{1}^{\prime}(t)= & \tilde{\lambda}_{1}^{\prime}(T) \exp \left\{i\left[\omega_{\lambda^{\prime}}^{*}(t-T)-\int_{T}^{t} \Delta_{1}^{*}\left(t^{\prime}\right) d t^{\prime}\right]\right\} \\
\tilde{\lambda}_{2}^{\prime}(t)= & \tilde{\lambda}_{2}^{\prime}(T) \exp \left\{i\left[\omega_{\lambda^{\prime}}^{*}(t-T)-\int_{T}^{t} \Delta_{2}^{*}\left(t^{\prime}\right) d t^{\prime}\right]\right\} \\
& \times \exp \left[\frac{1}{\hbar} \int_{T}^{t} V_{\mathrm{opt}}\left(t^{\prime}\right) d t^{\prime}\right],
\end{aligned}
$$

which leads in the limits (17) to

$$
\begin{gathered}
\tilde{\lambda}_{1}^{\prime}(0) \ll \tilde{\lambda}_{2}^{\prime}(0), \\
\tilde{\lambda}_{2}^{\prime}(0)=\tilde{\lambda}_{2}^{\prime}(T) \exp \left\{i\left[\omega_{\lambda^{\prime}}^{*}\left(T^{\prime}-T\right)-\int_{T}^{T^{\prime}} \Delta_{2}^{*}(t) d t\right]\right\} \\
\times \exp \left[+\frac{1}{\hbar} \int_{T}^{T^{\prime}} V_{\mathrm{opt}}(t) d t\right] .
\end{gathered}
$$

It corresponds to the Schrödinger equation,

$$
\left[-i \frac{\partial}{\partial t}+\left(\begin{array}{cc}
\Delta_{1}^{*}(t) & \Omega^{*}(t) \\
\Omega(t) & \Delta_{2}^{*}(t)
\end{array}\right)\right]\left(\begin{array}{c}
a_{1}^{\prime}(t) \\
a_{2}^{\prime}(t)
\end{array}\right)=0,
$$

with the initial condition $a_{1}^{\prime}(0)=0, a_{2}^{\prime}(0)=1$ for which we get

$$
a_{2}^{\prime}(T)=e^{i \int_{T}^{T^{\prime}} \Delta_{2}^{*}(t) d t} e^{-i \omega_{\lambda^{\prime}}^{*} T^{\prime}} \exp \left[-\frac{1}{\hbar} \int_{T}^{T^{\prime}} V_{\mathrm{opt}}(t) d t\right] .
$$

One can connect it to $a_{1}(T)$ as described in Appendix A, which induces

$$
\begin{aligned}
a_{1}(T)= & \exp \left\{-i \int_{0}^{T}\left[\Delta_{1}(t)+\Delta_{2}(t)\right] d t\right\} e^{-i \int_{T}^{T^{\prime}} \Delta_{2}(t) d t} \\
& \times e^{+i \omega_{\lambda^{\prime}} T^{\prime}} \exp \left[-\frac{1}{\hbar} \int_{T}^{T^{\prime}} V_{\mathrm{opt}}(t) d t\right]
\end{aligned}
$$

Identifying Eqs. (29) and (37) leads to

$$
\begin{aligned}
\operatorname{Im}\left(\omega_{\lambda^{\prime}}\right)= & \frac{1}{T^{\prime}}\left(\int_{0}^{T^{\prime}} \operatorname{Im}\left[\Delta_{2}\left(t^{\prime}\right)\right] d t^{\prime}+\int_{0}^{T} \operatorname{Im}\left[\Delta_{1}\left(t^{\prime}\right)\right] d t^{\prime}\right) \\
& -\frac{1}{T^{\prime}} \ln \left[\left|a_{1}(T)\right|\right]-\frac{1}{\hbar T^{\prime}} \int_{T}^{T^{\prime}} V_{\text {opt }}\left(t^{\prime}\right) d t^{\prime},
\end{aligned}
$$

which gives a relation between the imaginary parts of the two eigenvalues:

$$
\begin{aligned}
\operatorname{Im}\left(\omega_{\lambda^{\prime}}\right)= & -\frac{1}{\hbar T^{\prime}} \int_{T}^{T^{\prime}} V_{\mathrm{opt}}\left(t^{\prime}\right) d t^{\prime}-\operatorname{Im}\left(\omega_{\lambda}\right) \\
& +\frac{1}{T^{\prime}} \int_{0}^{T^{\prime}} \operatorname{Im}\left[\Delta_{1}\left(t^{\prime}\right)+\Delta_{2}\left(t^{\prime}\right)\right] d t^{\prime} .
\end{aligned}
$$

This central relation shows that the connected eigenvalue, in general, will be well isolated from the other one for a large enough area of the absorbing potential. More precisely, we have $-\operatorname{Im}\left(\omega_{\lambda^{\prime}}\right) \gg-\operatorname{Im}\left(\omega_{\lambda}\right)$ when

$$
\begin{aligned}
& \frac{1}{\hbar T^{\prime}} \int_{T}^{T^{\prime}} V_{\mathrm{opt}}\left(t^{\prime}\right) d t^{\prime} \gg-2 \operatorname{Im}\left(\omega_{\lambda}\right) \\
& +\frac{1}{T^{\prime}} \int_{0}^{T^{\prime}} \operatorname{Im}\left[\Delta_{1}\left(t^{\prime}\right)+\Delta_{2}\left(t^{\prime}\right)\right] d t^{\prime} .
\end{aligned}
$$

This feature will be useful in numerical calculations; in particular, it will improve the rate of convergence of the wave operator method [6] when applied to the location of the, thus, isolated connected eigenvalue.

However, the separation between the imaginary parts of eigenvalues can be absent in practice for specific cases of good population transfer. This is analyzed in Sec. V.

\section{NUMERICAL LIMITATIONS AND ACCURACY}

In this section, we study the numerical limitations of the method, restricting the discussion to the situation $c_{1}(0)=1, c_{2}(0)=0$. For simplicity, we consider the situation $\operatorname{Im}\left[\Delta_{2}(t)\right]=\operatorname{Im}\left[\Delta_{1}(t)\right]=0$.

\section{A. General cases}

The accuracy of the method can roughly be estimated from the imperfect initial connection with the eigenvector $|\lambda\rangle$, that is, from the small quantity $\lambda_{2}(0)$. In general, when $\lambda_{2}(T)$ and $\lambda_{1}(T)$ are of the same order, we obtain, for the error in the final amplitude, from Eq. (16),

$$
\left|a_{1}(T)-a_{1}^{(\mathrm{CATM})}(T)\right| \sim \exp \left[-\frac{1}{\hbar} \int_{T}^{T^{\prime}} V_{\mathrm{opt}}\left(t^{\prime}\right) d t^{\prime}\right],
$$

where $a_{1}^{(\mathrm{CATM})}(T)$ is the probability amplitude of state 1 at the end of the physical process obtained from the CATM method. This is shown to give a correct estimation of the accuracy of the method when it is tested numerically (see Sec. VI).

We remark that this estimation does not obviously take the grid size effect into account. This is studied numerically in Sec. VI.

\section{B. Case of good population transfer}

The estimation (41) is not valid when the population transfer at the end of the process is efficient: $\left|a_{1}(T)\right| \rightarrow 0$, since, in Eq. (16), we then have $\left|\lambda_{2}(T) / \lambda_{1}(T)\right| \gg 1$. The area of the optical potential should be large enough to satisfy the connectivity to a unique Floquet eigenvector: $\lambda_{2}(0) / \lambda_{1}(0) \rightsquigarrow$ 0, that is, from Eq. (19),

$$
\frac{1}{\hbar} \int_{T}^{T^{\prime}} V_{\mathrm{opt}}(t) d t \gg-\ln \left[\left|a_{1}(T)\right|\right] .
$$

One limiting case is when there is no separation between the imaginary parts of the eigenvalues,

$$
\operatorname{Im}\left(\omega_{\lambda^{\prime}}\right)=\operatorname{Im}\left(\omega_{\lambda}\right),
$$


leading, from Eqs. (30) and (38), to

$$
\frac{1}{\hbar} \int_{T}^{T^{\prime}} V_{\mathrm{opt}}\left(t^{\prime}\right) d t^{\prime}=-2 \ln \left[\left|a_{1}^{(\mathrm{CATM})}(T)\right|\right] .
$$

This equation shows that, in this case of equal quasienergies, the inequality (42) is satisfied with only a factor 2 . More precisely, we have

$$
\frac{\lambda_{2}(0)}{\lambda_{1}(0)} \approx \exp \left[-\frac{1}{2 \hbar} \int_{T}^{T^{\prime}} V_{\mathrm{opt}}(t) d t\right] .
$$

Thus, one can still satisfy $\lambda_{2}(0) / \lambda_{1}(0) \rightsquigarrow 0$ to get the connection to a unique Floquet eigenvector for good accuracy by imposing

$$
\frac{1}{2 \hbar} \int_{T}^{T^{\prime}} V_{\mathrm{opt}}(t) d t \gg 1
$$

Thus, this condition (46), a bit more restrictive than Eq. (17a) is sufficient to obtain quite good relative accuracy of the solution in the case of good population transfer, even if, in that case, the imaginary parts of the Floquet eigenvalues are close together.

We can use this limiting case (43) to estimate the absolute accuracy of the method. Assuming $\operatorname{Im}\left(\omega_{\lambda^{\prime}}\right) \leqslant \operatorname{Im}\left(\omega_{\lambda}\right)$, we get

$$
\left|a_{1}^{(\mathrm{CATM})}(T)\right| \geqslant \exp \left[-\frac{1}{2 \hbar} \int_{T}^{T^{\prime}} V_{\mathrm{opt}}\left(t^{\prime}\right) d t^{\prime}\right],
$$

that is, we cannot numerically obtain a population $\left|a_{1}^{(\mathrm{CATM})}(T)\right|^{2}$ of state 1 at the end of the physical process smaller than $\exp \left[-\frac{1}{\hbar} \int_{T}^{T^{\prime}} V_{\text {opt }}\left(t^{\prime}\right) d t^{\prime}\right]$, which, thus, gives a numerical limitation of the depopulation of the initial state.

\section{NUMERICAL INVESTIGATION}

The method is investigated numerically in this section through the examples of two- and three-state systems driven by a time-dependent field. For instance, they can correspond to atoms submitted to resonant laser pulses in the rotating wave approximation (RWA) [10,11].

\section{A. Some results for selected examples}

The first example is a two-state system $\{|1\rangle,|2\rangle\}$, which is subjected to a pulsed coupling of frequency little detuned with the transition frequency. The detuning is denoted as $\Delta$, and $\Omega$ is the coupling (Rabi frequency). In the dressed state picture of the RWA, the Hamiltonian is (in units such that $\hbar=1$ )

$$
H=\left(\begin{array}{cc}
0 & \Omega \\
\Omega & \Delta
\end{array}\right)=\left(\begin{array}{cc}
0 & \Omega_{0} \sin ^{2}\left(\frac{\pi t}{T}\right) \\
\Omega_{0} \sin ^{2}\left(\frac{\pi t}{T}\right) & \Delta_{0} \cos \left(\frac{\pi t}{T}+\phi_{0}\right)
\end{array}\right) .
$$

We will consider, as an initial condition, (i) $|\Psi(t=0)\rangle=|1\rangle$, from which we expect a final quasi-inversion of population for large enough $\Omega_{0} T$ and $\Delta_{0} T$ (adiabatic passage, see Refs. [10,11]), and (ii) the more complicated situation $|\Psi(0)\rangle=c_{1}|1\rangle+c_{2}|2\rangle$.

The second example is that of a three-level system $\{|1\rangle,|2\rangle,|3\rangle\}$ driven by two near-resonant laser fields with Rabi frequencies $\Omega_{p}$ and $\Omega_{s}$, tuned to the transitions $1 \leftrightarrow 2$ and $2 \leftrightarrow 3$, respectively. We allow a detuning $\Delta$ between the transition frequency $1 \rightarrow 2$ and the laser frequency and assume a two-photon resonance. The initial state is $|1\rangle$. Here, the RWA Hamiltonian takes the form

$$
H=\left(\begin{array}{ccc}
0 & \Omega_{p} & 0 \\
\Omega_{p} & \Delta & \Omega_{s} \\
0 & \Omega_{s} & 0
\end{array}\right) .
$$

We study two situations, on one hand, the intuitive case: We first turn on the coupling between levels 1 and 2, then between levels 2 and 3,

$$
\begin{gathered}
\Omega_{p}=\Omega_{0} \sin ^{2}\left(\frac{\pi t}{T_{1}}\right), \quad \forall t \in\left[0, T_{1}\right] \quad(0 \text { elsewhere }), \\
\Omega_{s}=\Omega_{0} \sin ^{2}\left(\frac{\pi t-T_{1} / 2}{T_{1}}\right), \quad \forall t \in\left[\frac{1}{2} T_{1}, \frac{3}{2} T_{1}\right], \\
\Delta=\Delta_{0} .
\end{gathered}
$$

With $\Omega_{0} T_{1}=20$ and $\Delta_{0} T_{1}=0$, we expect to observe oscillations without complete population exchange to state $|3\rangle$. With $\Delta_{0} T_{1}=20$, a partial transfer to $|3\rangle$ occurs with less oscillations.

On the other hand, the stimulated Raman adiabatic passage (STIRAP) case is exactly the inverse of the first configuration [12]:

$$
\begin{array}{cc}
\Omega_{p}=\Omega_{0} \sin ^{2}\left(\frac{\pi t-T_{1} / 2}{T_{1}}\right), & \forall t \in\left[\frac{1}{2} T_{1}, \frac{3}{2} T_{1}\right], \\
\Omega_{s}=\Omega_{0} \sin ^{2}\left(\frac{\pi t}{T_{1}}\right), & \forall t \in\left[0, T_{1}\right], \\
\Delta=0 . &
\end{array}
$$

With $\Omega_{0} T_{1}=20$ and $\Delta_{0} T_{1}=0$, STIRAP allows a large transfer of the population to $|3\rangle$.

Here, the total physical time interval $T$ is $3 / 2$ times the period $T_{1}$ of the sine function $[0, T]=\left[0,3 / 2 T_{1}\right]$; the additional time interval will begin at $3 / 2 T_{1}$ for a duration of $T_{1}$.

In the subsequent discussion, we use the labels (i) and (ii) for the two-level system with initial state $|1\rangle$ and the superposition of states, respectively. The labels (iii) and (iv) refer to the threelevel system in the intuitive or STIRAP situations, respectively.

\section{B. Calculating with CATM}

From a technical point of view, the calculation involves the five following steps:

(1) Construction of the matrix representation of the Floquet Hamiltonian (some details are given in Appendix B).

(2) Diagonalization of the Floquet matrix.

(3) Selection of $N$ Floquet eigenstates belonging to the first Brillouin zone (for a problem with $N$ levels).

(4) Detection of the appropriate connected Floquet eigenstate, i.e., corresponding to the smallest imaginary part of the eigenvalue in absolute value as a criterion.

(5) Production of the wave function via Eq. (5).

In principle, only one vector computation is needed. For our small-scale examples, we can easily use direct complete diagonalization. However, for larger systems, the time-dependent wave operator can be used to find the required eigenstate of the corresponding large matrix. 


\section{A comparison with direct integration}

We analyze the results obtained with the Floquet eigenstate that possesses the smallest value of $\left|\operatorname{Im}\left(\omega_{\lambda}\right)\right|$, as predicted by the theory. Next, we calculate the populations,

$$
p_{n}(t)=|\langle n \mid \Psi(t)\rangle|^{2},
$$

and the relative phases,

$$
\beta_{n}(t)=\arg [\langle n \mid \Psi(t)\rangle]
$$

for all the previously presented situations. We compare the CATM results for the population and phase with those of a direct integration using the propagation equation,

$$
|\Psi(t+\Delta t)\rangle=\exp \left[-i \hbar^{-1} H\left(t+\frac{\Delta t}{2}\right) \Delta t\right]|\Psi(t)\rangle,
$$

with $\Delta t$ as a sufficiently small time step. For the CATM calculation, the size of the Fourier basis set was $N=256$, which is ample for both stable computation and graphical representation.

\section{Two-state model}

For the two-level system, (i) the results are shown in Fig. 1. In frames (a-I) and (b-I), it is evident that, without the absorbing potential, the use of a single Floquet state is not sufficient. In frames (a-II) and (b-II), we can observe the effects of the absorbing potential. The initial populations approach $p_{1}(0)=1$ and $p_{2}(0)=0$, however, showing a small difference of a few percent from the reference calculation results. Phases begin to agree with those of the reference calculation, but the difference remains important, especially at the beginning. For the last case [(a-III) and (b-III)], one cannot detect any difference between the CATM and the reference results at the scale of the figure.

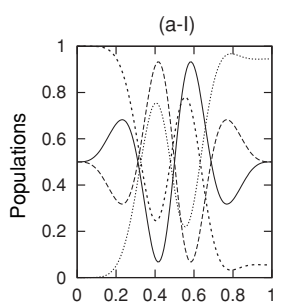

(b-l)
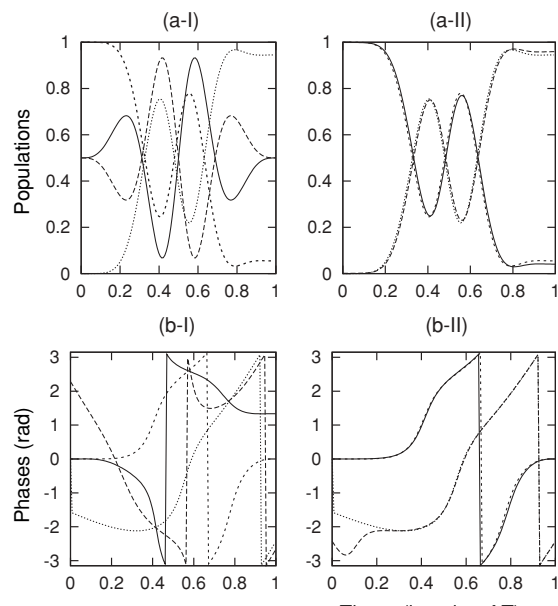

(b-II)
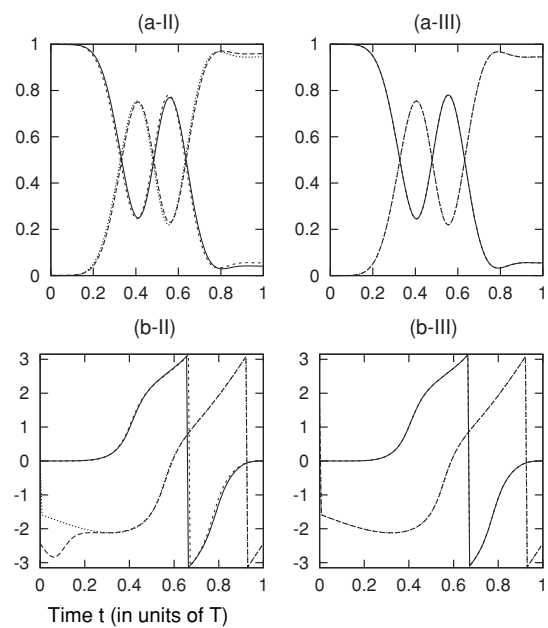

(b-III)

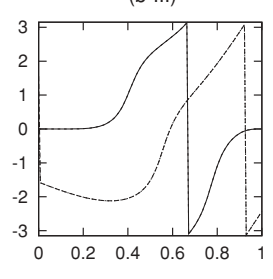

FIG. 1. Evolution of populations (a) and phases (b) for the twolevel system (i) with the initial state $|1\rangle$, and $\Omega_{0} T=10$ and $\Delta_{0} T=$ 10. Exact [i.e., numerical with the direct integration, cf. Eq. (54)] results [(short dashes) $p_{1}$ and $\beta_{1}$, (dots) $p_{2}$ and $\beta_{2}$ ] and CATM results [(solid line) $p_{1}$ and $\beta_{1}$, (long dashes) $p_{2}$ and $\beta_{2}$ ] for various amplitudes $V_{0}$ of the time-dependent absorbing potential: (I) $V_{0} T=0$, (II) $V_{0} T=$ 10 , and (III) $V_{0} T=40$.
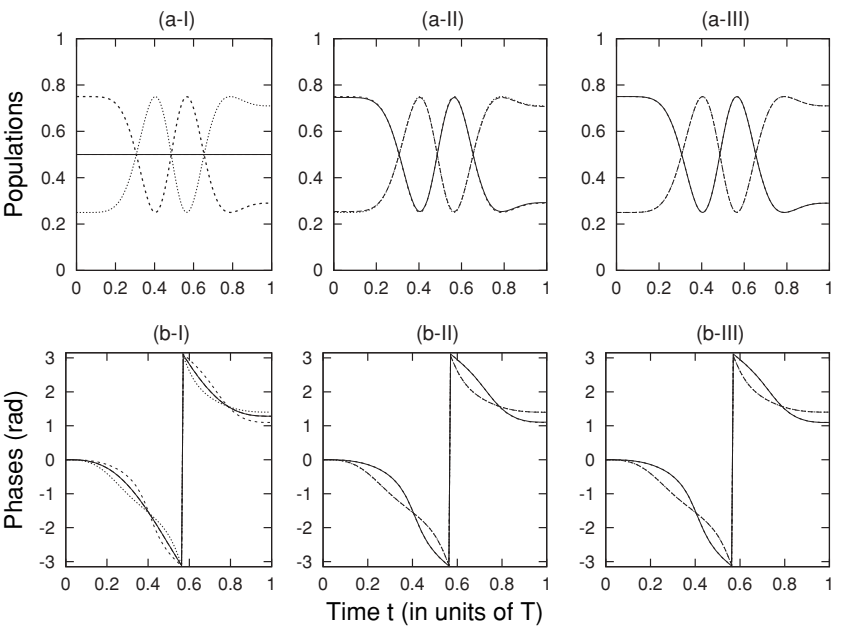

FIG. 2. Same as Fig. 1, but for the two-level system (ii), and $\Omega T=10, \Delta_{0} T=0$ with the initial state $\sqrt{0.75}|1\rangle+\sqrt{0.25}|2\rangle$ for various amplitudes $V_{0}$ of the time-dependent absorbing potential: (I) $V_{0} T=0$, (II) $V_{0} T=10$, and (III) $V_{0} T=40$.

Figure 2 shows the same quantities for the initial condition $|\Psi(0)\rangle=c_{1}|1\rangle+c_{2}|2\rangle, c_{1}=\sqrt{0.75}$, and $c_{2}=\sqrt{0.25}$. We have used the absorbing potential given by Eq. (11). The previous comments about the efficiency of the method remain valid. Figure 2 clearly illustrates the efficiency of the chosen matrix in reproducing the boundary conditions.

We now give a more precise analysis of how the exact solution is approached. To this end, we define a measure of the difference between the CATM results and the direct integration results. For the single component $\langle 1 \mid \Psi\rangle$ calculated by the two methods, we define the integrated difference of population and

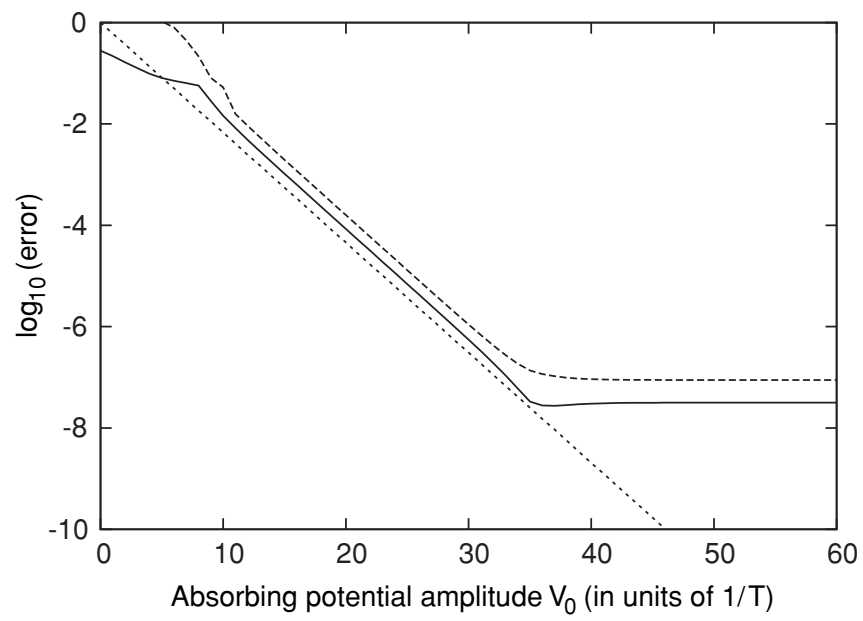

FIG. 3. Integrated logarithmic error estimation between direct integration and CATM with 512 time-grid points as a function of $V_{0}$, calculated with the first component $\langle 1 \mid \Psi\rangle$ for the two-level system (i). For errors on population, see Eq. (55a) (solid line), and for errors on angles, see Eq. (55b) (dashed line). We remark that these errors follow the anticipated exponential law Eq. (41) (dotted line) until they reach a plateau due to grid effects of CATM. 
(I)

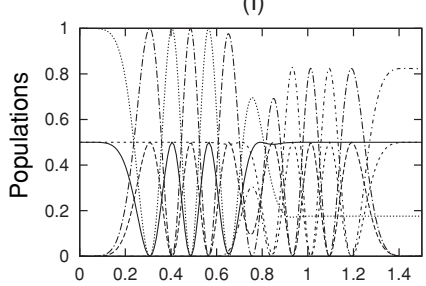

(III)

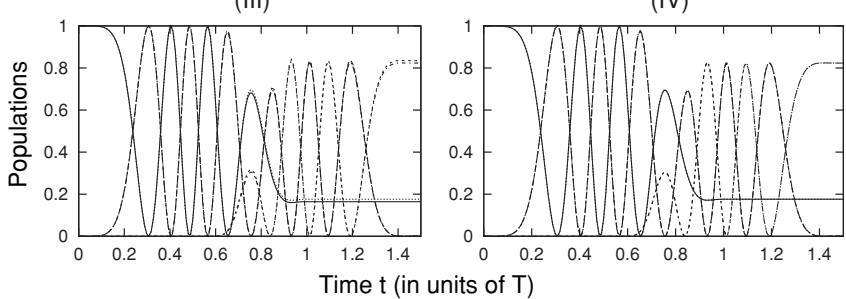

FIG. 4. Evolution of the populations $p_{n}(t)$ for the three-level model (iii); exact (numerical) results (dots) $p_{1}$, (long dot-dashes) $p_{2}$, (dot-dashes) $p_{3}$ and CATM results (solid line) $p_{1}$, (long dashes) $p_{2}$, and (short dashes) $p_{3}$ without detuning and for various amplitudes of the absorbing potentials, (I) $V_{0} T_{1}=0$, (II) 5 , (III) 10 , and (IV) 40 .

of angle

$$
\begin{aligned}
\epsilon_{p} & =\frac{1}{T} \int_{0}^{T}\left[\left|\langle 1 \mid \Psi(t)\rangle_{\mathrm{CATM}}\right|^{2}-|\langle 1 \mid \Psi(t)\rangle|^{2}\right] d t \\
\epsilon_{a} & =\frac{1}{T} \int_{0}^{T}\left\{\arg \left[\langle 1 \mid \Psi(t)\rangle_{\mathrm{CATM}}\right]-\arg [\langle 1 \mid \Psi(t)\rangle]\right\} d t .
\end{aligned}
$$

These quantities are represented in Fig. 3 as a function of the absorbing potential amplitude $V_{0}$. With the logarithmic scale, we observe a quasilinear law for $V_{0} \in[10,35]$ consistent with Eq. (41). The error estimates next reach plateaus that are interpreted by the grid effects due to the finite-basis representation of the time in the CATM method. Indeed, we can lower the level of the plateaus by increasing the number of the grid points (not shown).
(I)

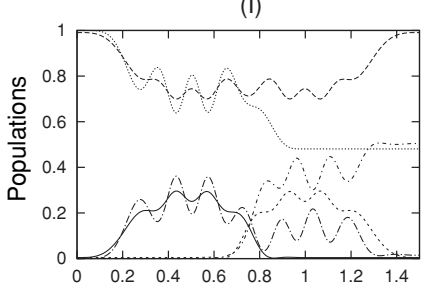

(III)

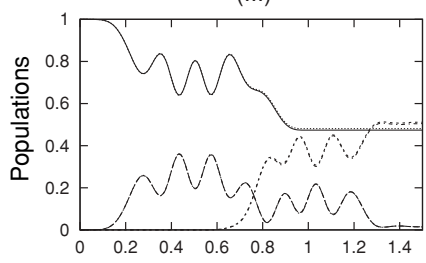

Time $t$ (in units of $T$ )
(II)

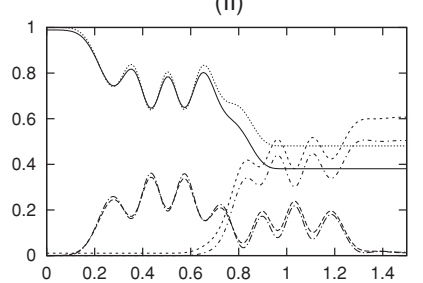

(IV)

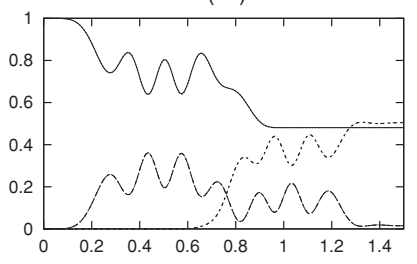

FIG. 5. Same as Fig. 4 but with a detuning $\Delta_{0} T_{1}=20$.
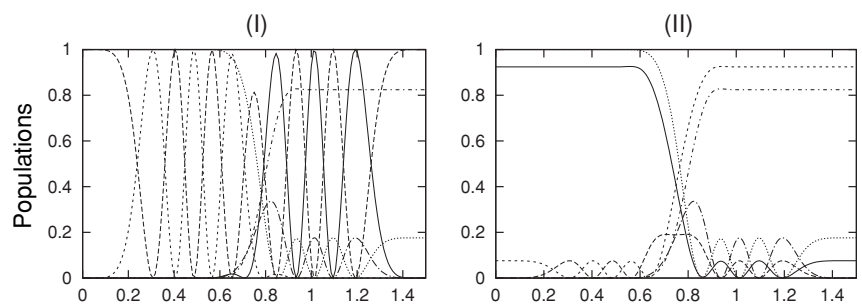

(III)

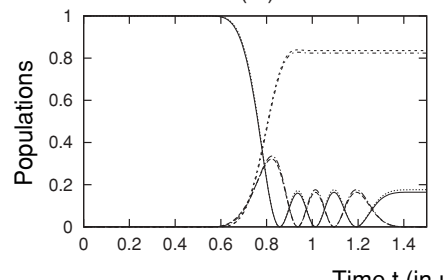

(IV)

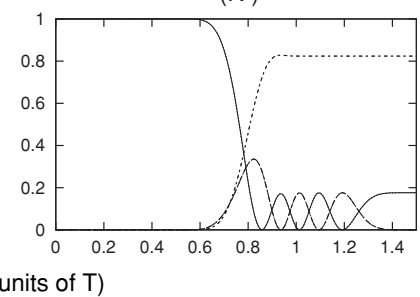

FIG. 6. Evolution of the populations $p_{n}(t)=|\langle n \mid \Psi(t)\rangle|^{2}$ in the STIRAP model (iv); exact (numerical) results (dots) $p_{1}$, (long dotdashes) $p_{2}$, (short dot-dashes) $p_{3}$ and CATM results (solid line) $p_{1}$, (long dashes) $p_{2}$, and (short dashes) $p_{3}$ without detuning and for various amplitude of the absorbing potential, (I) $V_{0} T_{1}=0$, (II) 5 , (III) 10 , and (IV) 40 .

\section{Three-state model}

For the three-level system, the evolution of the population in the three-level model (iii) (as defined in Sec. VI A) is shown in Figs. 4 and 5, without or with detuning $\left(\Delta_{0} T_{1}=0\right.$ or $\Delta_{0} T_{1}=$ 20). The selected field amplitude was $\Omega_{0} T_{1}=20$, and the absorbing potential was gradually turned on from $V_{0} T_{1}=0$ to $V_{0} T_{1}=40$. Here again, if the absorption is not sufficient, the results are wrong.

The results for the STIRAP model (iv) (as defined in Sec. VI A) are displayed in Fig. 6. The coupling terms between levels 2 and 3 are turned on before the coupling terms between 1 and 2, and a relatively large population inversion is observed. (a) $\operatorname{Re}\left(\omega_{\lambda}\right)$ vs $V_{0}$ (in units of $1 / T$ )

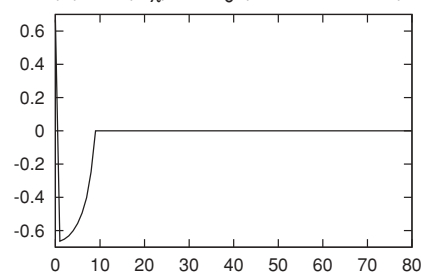

(c) $\operatorname{Re}\left(\omega_{\lambda^{\prime}}\right)$

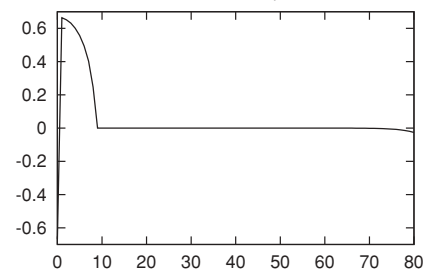

(b) $\operatorname{Im}\left(\omega_{\lambda}\right)$

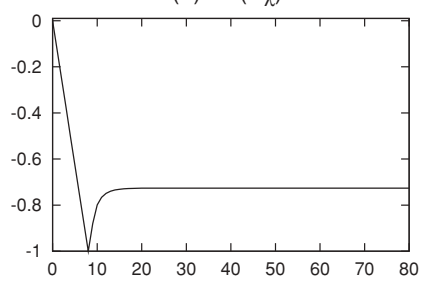

(d) $\operatorname{Im}\left(\omega_{\lambda^{\prime}}\right)$

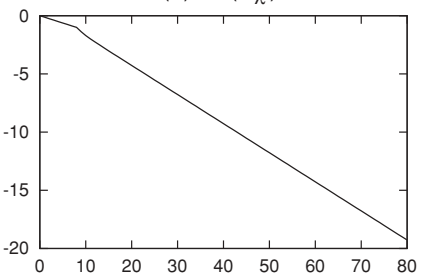

FIG. 7. (a) $\operatorname{Re}\left(\omega_{\lambda}\right)$, (b) $\operatorname{Im}\left(\omega_{\lambda}\right)$, (c) $\operatorname{Re}\left(\omega_{\lambda^{\prime}}\right)$, and (d) $\operatorname{Im}\left(\omega_{\lambda^{\prime}}\right)$ versus $V_{0}$ (all in units of $1 / T_{1}$ ) for the two-level model (i). When $V_{0}$ grows, $\omega_{\lambda^{\prime}}$ moves away from $\omega_{\lambda}$ acquiring an imaginary part proportional to $V_{0}$. 


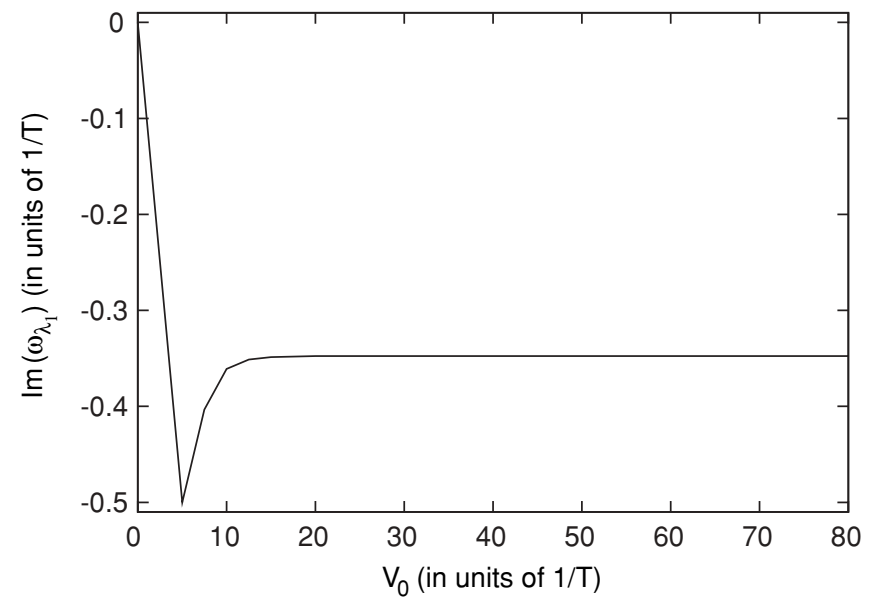

FIG. 8. $\operatorname{Im}\left(\omega_{\lambda_{1}}\right)$ versus $V_{0}$ for the three-level model (iii).

\section{The expansion of the spectrum}

We now analyze the effect of dilatation of the eigenvalues by the absorbing potential, that is, the feature of separating the connected eigenvalue with respect to the other ones. Figure 7 shows the Floquet eigenvalues $\left\{\omega_{\lambda}\right\}$ and $\left\{\omega_{\lambda^{\prime}}\right\}$ in the first Brillouin zone calculated for the two-level model (i) as functions of $V_{0}$. Apart from small absorbing potential amplitudes where one notices an ambiguity concerning the labeling of the eigenvalue [13], $\operatorname{Im}\left(\omega_{\lambda}\right)$ is a constant value in agreement with Eq. (30), and $\operatorname{Im}\left(\omega_{\lambda^{\prime}}\right)$ shows a linear evolution as predicted by Eq. (39).

Figures 8 and 9 refer to the three-level system (iii) and show the same features. Concentrating on the imaginary part of the connected Floquet eigenvalue (Fig. 8), we see that, after a region of stabilization, $\operatorname{Im}\left(\omega_{\lambda_{1}}\right)$ is no longer affected by the growth of the absorbing potential. In contrast, both $\operatorname{Im}\left(\omega_{\lambda_{2}}\right)$ and $\operatorname{Im}\left(\omega_{\lambda_{3}}\right)$ acquire imaginary parts that are linear with respect to $V_{0}$.

This feature will be useful in practice for large systems, in particular, if a wave operator method is used to find the Floquet eigenstate [6], since that method is efficient for finding isolated eigenvalues.

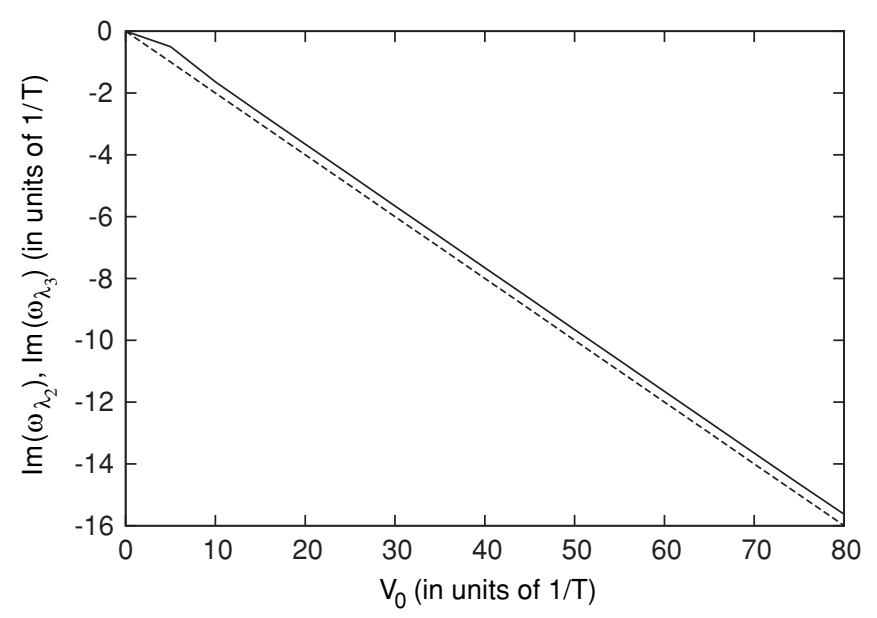

FIG. 9. (Solid line) $\operatorname{Im}\left(\omega_{\lambda_{2}}\right)$ and (dashed line) $\operatorname{Im}\left(\omega_{\lambda_{3}}\right)$ versus $V_{0}$ for the three-level model (iii).

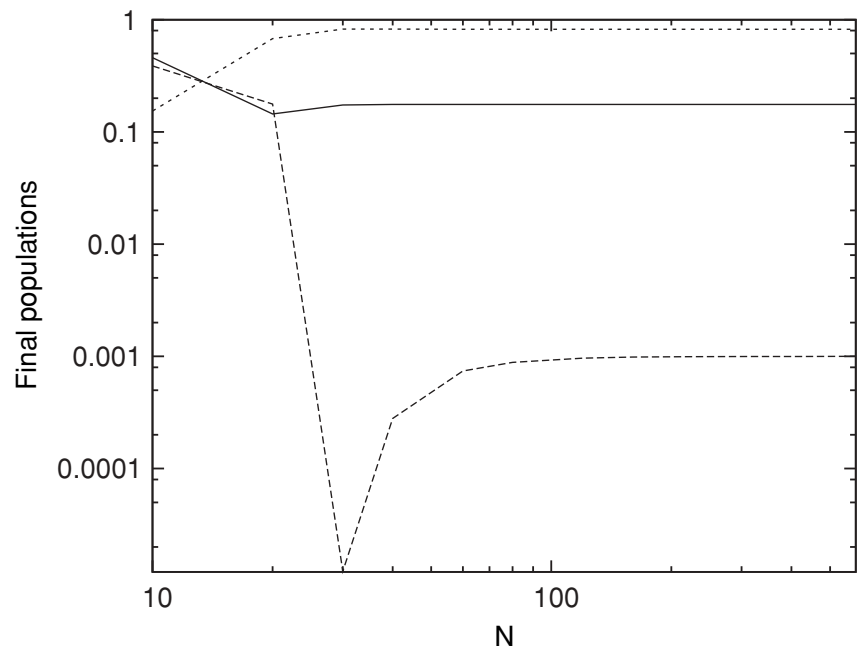

FIG. 10. Stability of final populations (solid line) $p_{1}$, (dashed line) $p_{2}$, and (dotted line) $p_{3}$ functions of the Fourier time-grid point number $N$ in logarithmic scale.

\section{E. The influence of the number of Fourier basis functions}

Here, we give some details about the stability of the results as the number of Fourier basis functions $N$ is reduced in the CATM calculation. To increase calculational speed and to decrease memory requirement, it appears necessary to use as small a value of $N$ as possible. Figure 10 shows how the final populations obtained in the CATM calculation vary as $N$ is increased. These calculations correspond to the STIRAP model (iv).

The values $p_{3} \simeq 0.82$ and $p_{1} \simeq 0.18$ are stable for $N \gtrsim 30$, but $p_{2} \simeq 10^{-3}$ is not obtained accurately until $N$ is about 80. We see that finding small probabilities in absolute values requires a more precise description of the temporal evolution; however, about 80 grid points appear to be ample for the calculations. The general principle is to choose an $N$ that is high enough to follow the time variations in the Hamiltonian and to obtain accurate values for small probabilities.

\section{CONCLUSION}

The optimum computational implementation of the CATM is still being actively researched, but the basic principles behind the method are simple to follow. A static absorbing potential is often used in treating the time development of a wave function within the Floquet formalism. The novelty of our approach is that the absorbing potential is given a time-dependent form such that it actively constrains the wave function, both by imposing the correct boundary conditions on it and by modifying the spectrum so that the specific eigenvalue that is appropriate to describe the dynamical process is rendered relatively isolated from the other eigenvalues. The dynamical problem is then rendered into an eigenvalue problem in which the isolated eigenvalue is easier to find by techniques, such as the Bloch wave operator method. That it is indeed possible to choose the time-dependent potential so as to produce the favorable features described above has been demonstrated for two small-scale systems for which accurate comparison results are available. For these small test systems, the CATM 
gives accurate results, although it is clear that the eigenvalue problems that arise can involve strongly non-Hermitian matrices.

The CATM has some formal advantages for systems with a time-dependent Hamiltonian. A common approach for such systems is to use a step-by-step propagation procedure with very small time steps. Thus, many time steps are required to cover a given time interval, and this leads to an accumulation of errors as the propagation proceeds. In contrast, in the CATM, the solution is global over the full time interval, and so there is no accumulation of errors; this feature is similar to that shown by the $\left(t, t^{\prime}\right)$ method [5]. As expected (and confirmed in this paper), the accuracy achievable within the CATM is governed by the ability to reproduce the initial conditions by suitably adjusting the time-dependent potential and by the use of a sufficiently dense Fourier time grid to describe any fast time variations contained in the Hamiltonian.

Our model calculations have also made the role of the timedependent absorbing potential in dilating the Floquet spectrum clear so that the dynamical problem of propagation within a Hilbert space of a given dimension can be converted to that of locating an isolated eigenvalue of a non-Hermitian matrix of much larger dimension. Thus, the difficulty of solving the dynamical problem is converted into the technical problem of devising efficient algorithms for large non-Hermitian matrices. In Ref. [7], a previous version of the CATM was successfully tested on a molecular system involving a few hundred states. At the moment, we believe that the task of isolating and then calculating the important dynamically relevant complex eigenvalue is probably not possible within the CATM for systems that are much larger than those treated in Ref. [7]; nevertheless, the method may be useful for some systems that cause difficulties for the usual propagation methods.

\section{ACKNOWLEDGMENTS}

We acknowledge the support from the French Agence Nationale de la Recherche (Project CoMoC), the European Marie Curie Initial Training Network Grant No. CA-ITN214962-FASTQUAST, and from the Conseil Régional de Bourgogne.

\section{APPENDIX A: PROPERTIES OF DISSIPATIVE PROPAGATORS}

We consider a traceless time-dependent dissipative Hamiltonian $H_{T}$, i.e., having complex diagonal elements (with negative imaginary parts) and being self-adjoint when it is restricted to its nondiagonal elements. It has the corresponding propagator $U_{H_{T}}\left(t, t_{0}\right): i \frac{\partial}{\partial t} U_{H_{T}}\left(t, t_{0}\right)=H_{T} U_{H_{T}}\left(t, t_{0}\right)$, and its adjoint $H_{T}^{\dagger}$ has the propagator $U_{H_{T}^{\dagger}}\left(t, t_{0}\right)$. They satisfy

$$
\operatorname{det}\left[U_{H_{T}}\left(t, t_{0}\right)\right]=\operatorname{det}\left[U_{H_{T}^{\dagger}}\left(t, t_{0}\right)\right]=1
$$

From the definition of the propagators, we obtain $\frac{\partial}{\partial t}\left[U_{H_{T}^{\dagger}}^{\dagger}\left(t, t_{0}\right) U_{H_{T}}\left(t, t_{0}\right)\right]=0$, that is,

$$
U_{H_{T}^{\dagger}}^{\dagger}\left(t, t_{0}\right) U_{H_{T}}\left(t, t_{0}\right)=\mathbb{1} .
$$

For the two-state case of the general Hamiltonian,

$$
H=\left(\begin{array}{cc}
\Delta_{1} & \Omega \\
\Omega^{*} & \Delta_{2}
\end{array}\right),
$$

in general, with complex $\Delta_{1}$ and $\Delta_{2}$ and time-dependent parameters $\Delta_{j} \equiv \Delta_{j}(t)$ and $\Omega \equiv \Omega(t)$, we first decompose it as a term proportional to identity and a traceless term,

$$
H=\frac{\Delta_{1}+\Delta_{2}}{2} \mathbb{1}+H_{T},
$$

with

$$
H_{T}=\left(\begin{array}{cc}
-\frac{\Delta_{2}-\Delta_{1}}{2} & \Omega \\
\Omega^{*} & \frac{\Delta_{2}-\Delta_{1}}{2}
\end{array}\right) .
$$

The propagator for $H$ reads

$$
U_{H}\left(t, t_{0}\right)=\exp \left\{-i \frac{1}{2} \int_{t_{0}}^{t} d t^{\prime}\left[\Delta_{1}\left(t^{\prime}\right)+\Delta_{2}\left(t^{\prime}\right)\right]\right\} U_{H_{T}}\left(t, t_{0}\right) .
$$

If $\Delta_{1}$ and $\Delta_{2}$ are real (nondissipative self-adjoint Hamiltonian), the propagator $U_{H_{T}}\left(t, t_{0}\right)$ is unitary and, thus, is of the form

$$
U_{H_{T}}\left(t, t_{0}\right)=\left(\begin{array}{cc}
a & -b^{*} \\
b & a^{*}
\end{array}\right) .
$$

In the general case of complex $\Delta_{1}$ and $\Delta_{2}$, this is not true anymore. We write the propagator as

$$
U_{H_{T}}\left(t, t_{0}\right)=\left(\begin{array}{ll}
a & c \\
b & d
\end{array}\right), \quad a d-b c=1 .
$$

For the adjoint of $H$,

$$
H^{\dagger}=\frac{\Delta_{1}^{*}+\Delta_{2}^{*}}{2} \mathbb{1}+H_{T}^{\dagger},
$$

the propagator writes

$U_{H^{\dagger}}\left(t, t_{0}\right)=\exp \left\{-i \frac{1}{2} \int_{0}^{t} d t^{\prime}\left[\Delta_{1}^{*}\left(t^{\prime}\right)+\Delta_{2}^{*}\left(t^{\prime}\right)\right]\right\} U_{H_{T}^{\dagger}}\left(t, t_{0}\right)$,

where $U_{H_{T}^{\dagger}}\left(t, t_{0}\right)$ connects with $U_{H_{T}}\left(t, t_{0}\right)$ as

$$
U_{H_{T}^{\dagger}}\left(t, t_{0}\right)=\left(\begin{array}{ll}
d^{*} & -b^{*} \\
-c^{*} & a^{*}
\end{array}\right) .
$$

These properties are used to obtain a link between two wave functions resulting from the two orthogonal initial states $\left(\begin{array}{l}1 \\ 0\end{array}\right)$ and $\left(\begin{array}{l}0 \\ 1\end{array}\right)$ and driven by $H$ and $H^{\dagger}$, respectively, i.e., $U_{H_{T}}\left(t, t_{0}\right)\left(\begin{array}{l}1 \\ 0\end{array}\right)=\left(\begin{array}{c}a \\ b\end{array}\right)$ and $U_{H_{T}^{\dagger}}\left(t, t_{0}\right)\left(\begin{array}{l}0 \\ 1\end{array}\right)=\left(\begin{array}{c}-b^{*} \\ a^{*}\end{array}\right)$.

\section{APPENDIX B: CONSTRUCTION OF THE FLOQUET HAMILTONIAN}

In this appendix, we give some details about the structure of the Floquet Hamiltonian. For the time dimension, we work with a discrete variable representation (DVR) $\left\{\left|t_{i}\right\rangle\right\}, i=$ $1, \ldots, N$, associated with a Fourier finite-basis representation. 
We show that the time-derivative operator can be expressed simply in the DVR basis: Let $I_{j}$ be a column vector of component $\delta_{i j}, i=1, \ldots, N$, then

$$
\left\langle t_{i}\left|-i \frac{\partial}{\partial t}\right| t_{j}\right\rangle=\mathrm{FFT}_{i}^{-1}\left(\begin{array}{c}
\omega_{1} \mathrm{FFT}_{1}\left(I_{j}\right) \\
\vdots \\
\omega_{N} \mathrm{FFT}_{N}\left(I_{j}\right)
\end{array}\right),
$$

where $\mathrm{FFT}_{i}$ represents the $i$ th fast Fourier transform component and $\omega_{i}$ is the Fourier angular frequency defined by

$$
\begin{gathered}
\omega_{n}=\frac{2 \pi}{T^{\prime}}(n-1), \quad 1 \leqslant n \leqslant \frac{N}{2}, \\
\omega_{n}=\frac{2 \pi}{T^{\prime}}(n-1-N), \quad \frac{N}{2}<n \leqslant N .
\end{gathered}
$$

Due to the periodicity, Eq. (B2) is equivalent to

$$
\omega_{n}=\frac{2 \pi}{T^{\prime}}(n-1), \quad 1 \leqslant n \leqslant N .
$$

The matrix representation of $-i \frac{\partial}{\partial t}$ is diagonal in the molecular basis, and $H(t)$ and $\mathcal{V}(t)$ are approximately diagonal in the DVR time basis. Consequently, the Floquet Hamiltonian for the two-level models (i) and (ii) with the initial conditions $c_{1}=1$ and $c_{2}=0$ is approximately represented in the $\{|1\rangle,|2\rangle\} \otimes\left\{\left|t_{i}\right\rangle\right\}$ basis by

$$
\left(\begin{array}{ccccc}
\partial_{t_{11}} & \Omega_{1} & \partial_{t_{12}} & 0 & \cdots \\
\Omega_{1} & \left(\partial_{t_{11}}+\Delta_{1}-i V_{1}\right) & 0 & \partial_{t_{12}} & \\
\partial_{t_{21}} & 0 & \partial_{t_{22}} & \Omega_{2} & \\
0 & \partial_{t_{21}} & \Omega_{2} & \left(\partial_{t_{22}}+\Delta_{2}-i V_{2}\right) & \\
\vdots & & & & \ddots
\end{array}\right)
$$

with

$$
\begin{gathered}
\partial_{t_{i j}} \equiv\left\langle t_{i}\left|-i \hbar \frac{\partial}{\partial t}\right| t_{j}\right\rangle, \\
\Omega_{i} \equiv \Omega\left(t_{i}\right), \quad \forall t_{i} \in[0, T] \quad(0 \text { elsewhere }), \\
\Delta_{i} \equiv \Delta\left(t_{i}\right), \quad \forall t_{i} \in[0, T] \quad(0 \text { elsewhere }), \\
-i V_{i} \equiv-i V_{\text {opt }}\left(t_{i}\right)=-i V_{0} \sin ^{2}\left(\frac{t_{i}-T}{T^{\prime}-T}\right), \\
\forall t_{i} \in\left[T, T^{\prime}\right] \quad(0 \text { elsewhere }) .
\end{gathered}
$$

This construction can be generalized directly to treat threelevel or larger systems.
[1] A. Assion, T. Baumert, M. Bergt, T. Brixner, B. Kiefer, V. Seyfried, M. Strehle, and G. Gerber, Science 282, 919 (1998).

[2] M. H. Beck, A. Jäckle, G. A. Worth, and H.-D. Meyer, Phys. Rep. 324, 1 (2000).

[3] J. H. Shirley, Phys. Rev. 138, B979 (1965).

[4] S. Guérin and H. R. Jauslin, Adv. Chem. Phys. 125, 147 (2003).

[5] U. Peskin and N. Moiseyev, J. Chem. Phys. 99, 4590 (1993).

[6] G. Jolicard and J. P. Killingbeck, J. Phys. A 36, R411 (2003).

[7] G. Jolicard, D. Viennot, and J. P. Killingbeck, J. Phys. Chem. A 108, 8580 (2004).
[8] C. Leforestier, R. Bisseling, C. Cerjan, M. Feit, R. Friesner, A. Guldberg, A. Hammerich, G. Jolicard, W. Karrlein, H.-D. Meyer, N. Lipkin, O. Roncero, and R. Kosloff, J. Comp. Phys. 94, 59 (1991).

[9] R. Kosloff, Dynamics of Molecules and Chemical Reactions (Dekker, New York, 1996), Chap. 5.

[10] B. W. Shore, Acta Phys. Slovaca 58, 243 (2008).

[11] L. P. Yatsenko, S. Guérin, and H. R. Jauslin, Phys. Rev. A 65, 043407 (2002).

[12] N. V. Vitanov, T. Halfmann, B. W. Shore, and K. Bergmann, Annu. Rev. Phys. Chem. 52, 763 (2001).

[13] D. Viennot, G. Jolicard, and J. P. Killingbeck, J. Phys. A 41, 145303 (2008) 